\title{
Patients resisted attempts to link smoking to their current medical problems in general practice consultations
}

Pilnick A, Coleman T. "I'll give up smoking when you get me better": patients' resistance to attempts to problematise smoking in general practice (GP) consultations. Soc Sci Med 2003;57:135-45.

\section{Q In general practice consultations, how do general practitioners (GPs) introduce and advise patients who smoke about quitting? How do patients respond to this advice?}

\section{DESIGN}

Interactional analysis of videotaped patient-GP consultations and semistructured interviews with GPs.

\section{SETTING}

General practices in an East Midlands County in the UK

\section{PARTICIPANTS}

47 consultations between 47 patients who were self reported regular or occasional smokers \{mean age 41 y, $74 \%$ women\}* and 29 different GPs. 39 GPs were also interviewed.

\section{METHODS}

42 GPs who responded to a postal questionnaire measuring attitudes towards discussing smoking with patients were recruited. Of the 538 video recorded consultations, 47 (between 47 different patients and 29 GPs) that mentioned smoking were chosen for further analysis. In addition, 39 semistructured interviews with the GPs were done. Data were analysed from a conversation analytic standpoint to assess ways in which antismoking advice was produced and managed.

\section{MAIN FINDINGS}

Interview data showed that GPs were aware of, and keen to avoid, the possibility of negative responses from patients regarding smoking. In 18 consultations a link was made between smoking and the presenting condition; 3 subcategories of linkage were identified. Raising smoking as a general problem for people in a particular circumstance. For example, a GP made a general link between smoking (as a contraindication) and use of a particular birth control pill. Upon discovering that the patient smoked, the GP suggested changing the prescription (presenting issue) rather than that the patient quit smoking. The presenting issue was problematised rather than smoking. Smoking as a specific problem for a person in a specific circumstance. For example, after checking that a patient still smoked, a GP specifically linked smoking to the patient's condition (eg, asthma) and went on to problematise smoking in terms of increased expense and exacerbations. Patient responses were to downplay the cost or amount smoked, or to dissociate smoking from their condition: "I'll give up smoking when you get me better." Discussion topics were subsequently diverted from smoking. Worst case scenarios: stopping smoking as the solution to a specific (and potentially fatal) problem. For example, a GP responded to a patient's report of cough by directly suggesting that quitting smoking would resolve the

For correspondence: Dr A Pilnick, University of Nottingham, Nottingham, UK. alison.pilnick@nottingham.ac.uk

Source of funding: not stated. condition. The patient recounted previous attempts and difficulties trying to stop, acknowledging the undesirability of smoking. However, patients sometimes responded to worst case scenarios by becoming extremely defensive, giving contradictory responses, and minimising the smoking habit.

\section{CONCLUSIONS}

In general practice consultations, general practitioners raised and problematised smoking by linking it to patients' current medical conditions. However, patients resisted this linkage through justification and minimisation of the associated risks.

*Information provided by author.

\section{Commentary}

The study by Pilnick and Coleman offers an intriguing glimpse into the interactional dynamics that occur between GPs and their patients in the context of smoking cessation advice. Interestingly, this glimpse appears to challenge both existing research in this area and conventional practice wisdom suggesting that patients are more likely to take smoking cessation advice to heart when GPs personalise smoking by linking it to presenting health problems. Conversely, patients in this study became defensive about their smoking habit and resisted GPs' attempts to problematise smoking in terms of their presenting health concerns.

Conversational analysis is a useful method for exploring patientprovider interactions, as well as the more complex relationships these interactions reflect. From a practitioner's perspective, understanding the interactional dynamics depends on a deeper understanding of the nature of each patient-provider relationship. Are these relationships characterised by respect, trust, and reciprocity? "I'll give up smoking when you get me better" suggests that issues of control and power may exist in these relationships that are inconsistent with accepted models of effective relationships in preventive healthcare, wherein patients are equal and responsible partners, working in collaborative relationships with their health providers.

In their conclusions, the authors recognised that, like advice concerning other lifestyle choices that affect health, the provider's role in offering preventive health advice is subtly and qualitatively different from his or her role in curative care. Successful uptake and "buy in" of preventive or promotive health advice may depend more on the nature and trajectory of the patient-provider relationship itself, rather than the content or timing of specific interactions. Building effective relationships with patients is a complex and poorly understood skill that demands further consideration from practitioners and researchers alike.

Denise S Tarlier, RN, MSN, FNP-C (AANP) University of British Columbia Vancouver, British Columbia, Canada 